\title{
The validity of the reinstatement model of craving and relapse to drug use
}

Published online: 3 June 2003

(C) Springer-Verlag 2003

\section{Psychopharmacology (2003) \\ DOI 10.1007/s00213-003-1441-y}

Under the heading "Formal equivalence models," the second sentence should read: "These models are most often developed to simulate a specific critical sign or symptom of the human disorder, but are typically extended beyond mere formal similarity to assume characteristics of other types of models (Carlton 1983; McKinney 1989)."

The heading for the section that immediately follows the section entitled "Correlational models" should be "Functional equivalence models."
The online version of the original article can be found at http:// dx.doi.org/10.1007/s00213-003-1441-y

J. L. Katz (®)

Psychobiology Section, NIDA Addiction Research Center,

NIH DHHS,

Baltimore, MD 21224, USA

e-mail: jkatz@intra.nida.nih.gov

Tel.: +1-410-5501533

\section{S. T. Higgins}

Departments of Psychiatry and Psychology,

University of Vermont,

Burlington, VT 05401, USA
Under the heading "Functional equivalence models," the first sentence should read: "McKinney (1989) discussed models developed to evaluate etiological theories, and mechanistic models developed to study underlying mechanisms."

In the second paragraph under the heading "Assessments of studies of re-exposure to drug-paired stimuli", the second sentence from the end of the paragraph should read "One possible difference between the animal and human studies is the dependent variables." 